\title{
Article \\ The Compatibility of Three Silicone Oils with Polydimethylsiloxane and the Microstructure and Properties of Their Composite Coatings
}

\author{
Yuguo Jiang, Zhanping Zhang * and Yuhong Qi \\ Department of Materials Science and Engineering, Dalian Maritime University, Dalian 116026, China; \\ 1120180332jyg@dlmu.edu.cn (Y.J.); yuhong_qi@dlmu.edu.cn (Y.Q.) \\ * Correspondence: zzp@dlmu.edu.cn
}

check for

updates

Citation: Jiang, Y.; Zhang, Z.; Qi, Y. The Compatibility of Three Silicone Oils with Polydimethylsiloxane and the Microstructure and Properties of Their Composite Coatings. Polymers 2021, 13, 2355. https://doi.org/ $10.3390 /$ polym 13142355

Academic Editors: Hsiu-Wen Chien, Ying-Nien Chou and Dave

Mangindaan

Received: 16 May 2021

Accepted: 13 July 2021

Published: 18 July 2021

Publisher's Note: MDPI stays neutral with regard to jurisdictional claims in published maps and institutional affiliations.

Copyright: (c) 2021 by the authors. Licensee MDPI, Basel, Switzerland. This article is an open access article distributed under the terms and conditions of the Creative Commons Attribution (CC BY) license (https:// creativecommons.org/licenses/by/ $4.0 /)$.

Keywords: silicone; coating; silicone oil; compatibility; polydimethylsiloxane

\section{Introduction}

Marine biological fouling is one of the most common fouling forms in the ocean [1,2], which has caused great harm and potential safety hazard to ships, bridges, offshore drilling platforms and other offshore industrial facilities [3,4]. It is an effective and long-standing method to an apply antifouling coating on the surface of the metal immersed in seawater that needs to be protected [5]. It can not only inhibit the attachment of marine pollutants such as algae, oysters and even tiny bacteria and reduce the harm of marine biological pollution and but also effectively improve the life of ships and industrial facilities $[3,6]$. Organotin self-polishing antifouling coatings were widely used because of their excellent properties [7]. However, research found that the organotin antifouling coatings in use caused pollution to the ocean, damaging the marine ecological environment. With the promulgation and implementation of the "International convention for the control of harmful antifouling systems on ships" (AFC), many countries have turned to study environmentally friendly marine antifouling coatings $[8,9]$.

Silicone coatings can effectively prevent fouling organisms from adhering to the coating surface [10]. Due to its low surface energy and low elastic modulus, most of the mucus secreted by fouling organisms cannot wet the coating surface, meaning it is difficult for it to be fixed on the coating surface [11,12]. The lower elastic modulus enables the fouling organisms on the coating surface to peel off from the surface with lower energy under the scouring of seawater [13], which can effectively remove the attached organisms by the scouring effect of the ship and seawater during navigation [14], which is an efficient "fouling release" coating [15].

Organosilicon was first used in marine antifouling coatings in 1972, developed by Mueller [16]. However, it was not widely used due to the shortcomings of its poor mechanical properties, construction and recoating property. After 1990, Yonehara and colleagues 
produced a series of organosilicon coatings with different compositions and additives. Through panel immersion tests, they proved that the antifouling effect of silicone antifouling coatings was improved compared with traditional antifouling coatings [17]. After that, Masato, Takafumi and colleagues made different modifications to silicone antifouling coatings and achieved a good antifouling effect $[18,19]$. Ba et al. improved the mechanical properties of silicone coatings by adding different nano-powders [20].

Ships that stay in the port for a long time tend to collect and deface organisms on the surface [21]. The antifouling properties are often improved by adding silicone oil to silicone coatings to mimic the properties of silicone oil deposited on the skins of large animals in the ocean [22,23]. Moreover, the low toxicity of silicone oil poses little risk to marine life [24]. The earliest study on silicone oil-modified silicone coatings was in 1977; Milne tried to add inert silicone oil into silicone coatings to improve the antifouling performance [25]. Hoipkemeier-Wilson et al. added methyl silicone oil to silicone coatings and proved that adding silicone oil improved the antifouling performance through a spore adhesion experiment [26]. Truby et al. added phenyl silicone oil to silicone coatings and conducted long-term panel immersion tests in Hawaii; the results showed that the addition of silicone oil effectively reduced the adhesion strength of barnacles and oysters to the silicone coating [27].

During recent years, some research progress has been made on adding silicone oil to improve antifouling performance [28-30]. Research on mixing silicone oil into silicone coatings mainly focuses on the antifouling performance of some specific fouling organisms [31,32]. Ba et al. further expounded the influence of the type, viscosity and addition amount of silicone oil on the precipitation property of silicone oil on the surface [33]. Related studies were mainly focused on the antifouling performance of silicone coatings. However, the influence of the compatibility of silicone oil and silicone blend solutions on the structure of the blend coating is still unknown. This paper will focus on comparing the compatibility of three types of silicone oil with PDMS through thermodynamic calculation and contrast observations of the blend solution, and the influence of the compatibility on the mechanical properties and surface properties of the composite coatings containing solid and liquid phases.

\section{Materials and Methods}

\subsection{Material}

Hydroxy-terminated polydimethylsiloxane (PDMS) was purchased from Shandong Dayi Chemical Industry Co., Ltd. (Yantai, China), with a viscosity of 10,000 Pa.s and molecular weight of 60,000. Methyl silicone oil (MSO) was purchased from Shandong Dayi Chemical Industry Co., Ltd. (Yantai, China), with a viscosity of $15 \mathrm{~Pa} \cdot \mathrm{s}$ and a molecular weight of 1700. Phenyl silicone oil (PSO) was purchased from Shanghai Hualing Resin Co., Ltd. (Shanghai, China), with a viscosity of $30 \mathrm{~Pa} \cdot \mathrm{s}$ and a molecular weight of 450 . Methyl fluoro silicone oil (FSO) was purchased from Wuhan Huaxiang Kejie Biotechnology Co., Ltd. (Wuhan, China), with a viscosity of $1000 \mathrm{~Pa} \cdot \mathrm{s}$ and a molecular weight of 26,000. Ethyl orthosilicate (TEOS) was obtained from Tianjin Chemical Co., Ltd. (Tianjin, China). New bismuth decanoate (BIND) was obtained from Shanghai Deyin Chemical Co., Ltd. (Shanghai, China). Xylene and ethyl acetate were also analytical grade and provided by Yongda Chemical Reagents Co., Ltd. (Tianjin, China).

\subsection{Preparation of Coating Sample}

Generally, silicone paint consists of three parts: film former, crosslinking agent and catalyst. The film former used in the experiment is hydroxyl-terminated PDMS, which needs to be modified by adding silicone oil (silicone oil does not participate in the filmforming reaction).

Silicone paint with silicone oil was prepared by a two-step method: First, $200 \mathrm{~g}$ PDMS, and silicone oil with the corresponding mass ratio were added into a $500 \mathrm{~mL}$ metal stirring tank. The mixture was dispersed for $30 \mathrm{~min}$ at $3000 \mathrm{rpm}$ by using a sand mill 
dispersion mixer. After the dispersion, the mixture was poured into a clean tinplate tank for standing and defoaming to form component A. TEOS and xylene were mixed into a solution according to the mass ratio of 3:7, poured into a clean white plastic bottle and recorded as component $\mathrm{B}$. BIND and ethyl acetate were mixed into a solution according to the mass ratio of $3: 7$ and poured into a clean white plastic bottle, which was marked as component $\mathrm{C}$. The three components, $\mathrm{A}, \mathrm{B}$ and $\mathrm{C}$, were mixed according to the mass ratio of 20 (PDMS): 4:1 and stirred with a paint mixing knife for $3 \mathrm{~min}$, followed by painting and curing to obtain the composite coating.

The blank control sample without PSO was set as P0. The coating samples were set as $\mathrm{X}-\mathrm{ZY}$, in which the letter $\mathrm{X}$ is the type of silicone oil, where MSO represents methyl silicone oil (MSO), PSO represents phenyl silicone oil (PSO) and FSO represents fluoro silicone oil (FSO); the letter $\mathrm{Z}$ is the ratio $(\mathrm{O} / \mathrm{B})$ of silicone oil to base material, and the letter $\mathrm{Y}$ represents the mass of silicone oil per $100 \mathrm{~g}$ of PDMS.

\subsection{Characterization}

\subsubsection{Phase Separation of Silicone Oil/PDMS Blend Solution}

PDMS was blended with silicone oil at a mass ratio of $10 \%, 20 \%, 30 \%$ or $40 \%$ to $90 \%$. After dispersing for $10 \mathrm{~min}$ with a multi-purpose mixer at the speed of $3000 \mathrm{rpm}$, the mixture was poured into the test tube and kept for $48 \mathrm{~h}$. The phase separation state was observed and recorded by taking a photo. The blend solution samples were set as $\mathrm{X} \% \mathrm{Y} / \mathrm{PDMS}$, in which the letter $\mathrm{X}$ is the mass of silicone oil per $100 \mathrm{~g}$ of PDMS, and the letter $\mathrm{Y}$ is the type of silicone oil, where MSO represents methyl silicone oil (MSO), PSO represents phenyl silicone oil (PSO) and FSO represents fluoro silicone oil (FSO).

\subsubsection{Microscopic Observation of Silicone Oil/PDMS Blend Solution}

A small amount of the prepared mixed solution absorbed by the pipette was dripped on the glass slide with about $0.2 \mathrm{~g}$ solution, which was allowed to stand for $48 \mathrm{~h}$ to make the mixed solution fully spread on the surface of the glass slide. Then, the sample was observed with an optical microscope (OM), and photos were taken. The mixture samples were set as $\mathrm{XY}$, in which the letter $\mathrm{X}$ is the type of silicone oil, where MSO represents methyl silicone oil (MSO), PSO represents phenyl silicone oil (PSO) and FSO represents fluoro silicone oil (FSO); the letter $\mathrm{Y}$ is the mass of silicone oil per $100 \mathrm{~g}$ of PDMS.

\subsubsection{Fracture Observation of the Coatings}

The coating was cut into $40 \times 10 \mathrm{~mm}^{2}$ samples. They were immersed in liquid nitrogen and cooled for $20 \mathrm{~min}$ before breaking. The fracture morphology was observed by an Olympus OLS4000 confocal laser scanning microscope (CLSM) (Olympus (China) Co, Ltd, Beijing, China).

\subsubsection{Microscopic Surface Characterization}

The surface of the coatings with different silicone oils was observed by a WT-1000GM stereomicroscope (Shanghai micro path photoelectric technology, Shanghai, China) and by CLSM, photographed. The photos were processed by Photoshop software (Adobe Systems, San Francisco, CA, USA) to obtain the ratio of the silicone oil coverage area and coating coverage area.

\subsubsection{Mechanical Properties of the Coatings}

The tensile curve of the coating was measured using a UTM5105 computer-controlled electronic universal testing machine (Jinan Wance Testing Electric Equipment Co., Ltd., Jinan, China), with a tensile rate of $50 \mathrm{~mm} / \mathrm{min}$. The dumbbell-shaped sample was prepared according to the national standard, GB/T 528-2009 (ISO 37:2005 [34]); the length of the sample was $75 \mathrm{~mm}$, the gauge length was $25 \mathrm{~mm}$ and the width was $4 \mathrm{~mm}$. The tensile data less than $0.1 \mathrm{~mm} / \mathrm{mm}$ were selected to fit the elastic modulus of the coating. Three samples were prepared and tested for each coating. The stress-strain curve was plotted. 
The sample was placed on the surface of the operating table, and the TH2200 shore hardness tester (Beijing Shidai Zhifeng Technology Co., Ltd. Beijing, China) was held to make the presser foot parallel to the surface of the sample. The presser needle was pressed into the sample vertically without vibration. When the hardness tester made contact with the sample surface, it read and recorded the data within $1 \mathrm{~s}$, selecting 6 points for each sample, taking the average value as the hardness. To meet the test requirements, the thickness of the sample should be more than $5 \mathrm{~mm}$.

\subsubsection{Surface Properties of Coatings}

The contact angles of the coatings were measured by the angle measuring method. A JC2000C contact angle measuring instrument (China Zhongchen Co., Ltd., Shanghai, China) was used to extrude about $3 \mu \mathrm{L}$ of distilled $\mathrm{H}_{2} \mathrm{O}$ and $\mathrm{CH}_{2} \mathrm{I}_{2}$, and the camera of the equipment was used to save the image and record the outline of the contact angle between the droplet and the surface. For every coating, five positions were measured, and the average value was taken as the contact angle. Based on the measured water and diiodomethane contact angle (CA), the surface energy of the sample was calculated by the Owens double liquid method.

\section{Results}

\subsection{Phase Separation Thermodynamic of Silicone Oil/PDMS Mixture}

The chemical structures of three types of silicone oils, MSO, PSO and FSO, are shown in Figure 1. The different chemical structures determine their difference with PDMS, and the different compatibilities of each silicone oil and PDMS mixture.
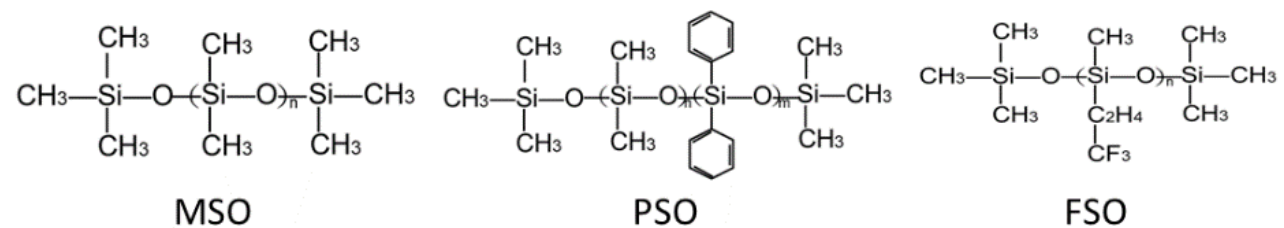

Figure 1. The structural formula of three types of inert silicone oils.

The Flory-Huggins lattice model is most commonly used to describe the mixing phenomenon of polymer solutions. Based on this model $[35,36]$, therefore, the thermodynamics of the silicone oil/PDMS blend solutions can be analyzed as follows.

Taking silicone oil as polymer A and PDMS as polymer B, we state that the molecular chain of polymers A and B contains "chain segments" $x_{A}$ and $x_{B}$, respectively. The amount of each substance in the silicone oil/PDMS mixture is $\mathrm{n}_{\mathrm{A}}$ and $\mathrm{n}_{\mathrm{B}}$, and the volume fraction is $\varphi_{\mathrm{A}}$ and $\varphi_{\mathrm{B}}$, respectively. The molar volume of both "chain segments" $\mathrm{A}$ and $\mathrm{B}$ is Vu. The total volume of the mixture system is $\mathrm{V} . \chi_{1}$ is the interaction parameter between the silicone oil and PDMS. The unit mixed Gibbs free energy $\Delta G_{M}$ of the mixture can be calculated by formula (1) [35].

$$
\Delta \mathrm{G}_{\mathrm{M}}=\mathrm{RTV}\left(\varphi_{\mathrm{A}} \ln \varphi_{\mathrm{A}} / \mathrm{x}_{\mathrm{A}}+\varphi_{\mathrm{B}} \ln \varphi_{\mathrm{B}} / \mathrm{x}_{\mathrm{B}}+\chi_{1} \varphi_{\mathrm{A}} \varphi_{\mathrm{B}}\right) / \mathrm{Vu}
$$

For a blend solution, we usually observe the phase separation point of the blend solution by an observation method and then substitute the data into Equation (1) to obtain the interaction parameters of the blend solution. However, by combining thermodynamics with the Flory-Huggins lattice model, we obtain the relationship between the interaction parameter $\chi_{1}$ and the solubility parameter of silicone oil $\delta_{1}$ and PDMS $\delta_{2} ; \mathrm{V}_{\mathrm{M} 1}$ is the molar volume of silicone oil [36].

$$
\mathrm{X}_{1}=\mathrm{V}_{\mathrm{M} 1}\left(\delta_{1}-\delta_{2}\right)^{2} / \mathrm{RT}
$$

The solubility parameter of PDMS $\delta_{2}$ was determined by the intrinsic viscosity method in this investigation, and it is equal to $15\left(\mathrm{~J} / \mathrm{cm}^{3}\right)^{1 / 2}$. Therefore, the solubility parameter 
of the silicone oil $\delta_{1}$ obtained by the group contribution method was substituted into Equation (2), and the interaction parameter $\chi_{1}$ between silicone oil and PDMS was obtained. As the main chain structure of silicone oil and PDMS is the same, their repeating unit is a $\mathrm{Si}-\mathrm{O}$ bond, the repeating unit can be regarded as a "chain segment" and the "chain segment" number of the polymer can be estimated by the relative molecular weight of the polymer. The molecular weight of PDMS resin is 60,000 , and the number of chain segments is 810 . The thermodynamic parameters calculated are listed in Table 1.

Table 1. The thermodynamic parameters of silicone oil/PDMS.

\begin{tabular}{cccc}
\hline Types of Silicone Oil & MSO & PSO & FSO \\
\hline$\chi_{1}$ & 0.0074 & 0.8118 & 71.7798 \\
$\delta_{1} /\left(\mathrm{J} / \mathrm{cm}^{3}\right)^{1 / 2}$ & 15.10 & 17.22 & 15.83 \\
$\mathrm{x}_{\mathrm{A}}$ & 22 & 6 & 351 \\
$\mathrm{~V}_{\mathrm{M} 1} /(\mathrm{mL} / \mathrm{mol})$ & 1770.83 & 432.69 & $17,894.00$ \\
\hline
\end{tabular}

Substituting the data in Table 1 into Equation (1), the curves of the concentration $\varphi_{\mathrm{A}}$ and $\Delta \mathrm{G}_{\mathrm{M}}$ of the mixture system of silicone oil and PDMS were obtained, as shown in Figure 2. According to thermodynamics, when $\Delta \mathrm{G}_{\mathrm{M}}<0$, the blend solution can spontaneously fuse, and when $\Delta \mathrm{G}_{\mathrm{M}}>0$, phase separation will inevitably occur. As it is shown in Figure 2a, for the mixed solution of methyl fluoro silicone oil and PDMS at any concentration, $\Delta \mathrm{G}_{\mathrm{M}}>0$, and this means that methyl fluoro silicone oil and PDMS cannot be miscible, and phase separation between methyl fluoro silicone oil and PDMS is inevitable. On the contrary, as shown in Figure 2a,b, when methyl silicone oil and PDMS are blended, $\Delta G_{M}$ is always less than 0 at any ratio, that is to say, methyl silicone oil and PDMS are miscible, and phase separation cannot occur. As it is shown in Figure $2 b$, when the concentration of phenyl silicone oil and PDMS is low, $\Delta \mathrm{G}_{\mathrm{M}}<0$, and then it is more than $1.9 \%, \Delta \mathrm{G}_{\mathrm{M}}>0$, which means that when the volume concentration of phenyl silicone oil reaches $1.9 \%$, phase separation begins to appear. In other words, the larger the interaction parameter $\chi_{1}$ between silicone oil and polydimethylsiloxane, the lower the compatibility between silicone oil and polydimethylsiloxane, and the easier it is for phase separation to occur.
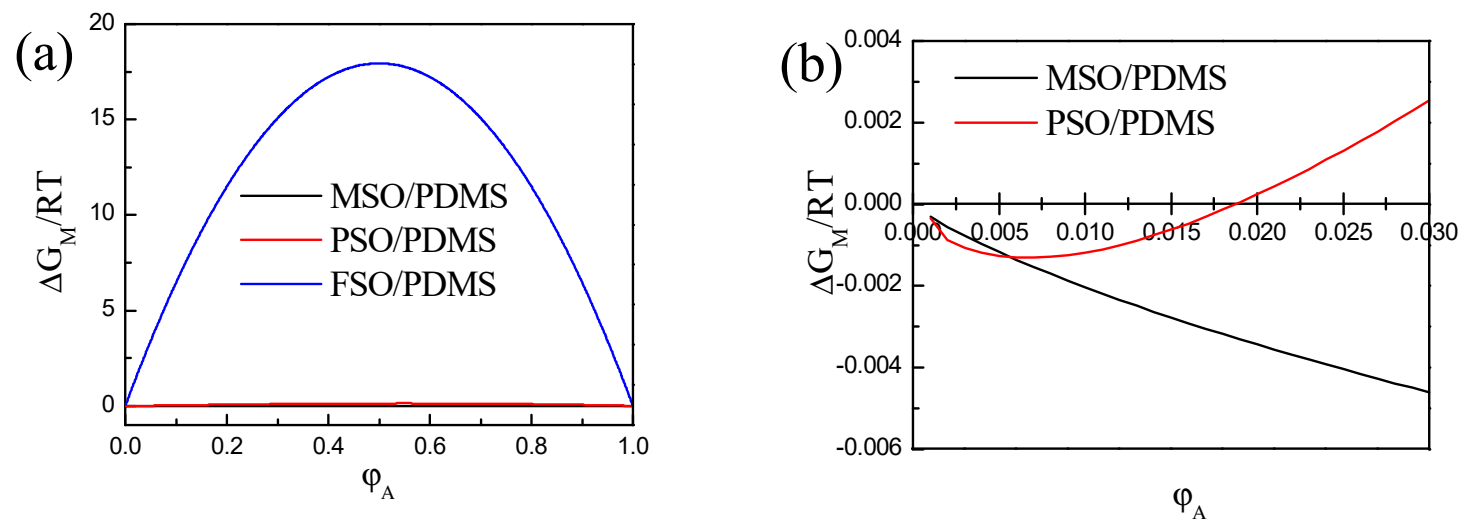

Figure 2. Mixed Gibbs free energy of three silicone oil with PDMS blends: (a) whole concentration range; (b) local amplification for low concentrations.

\subsection{Experimental Verification of Silicone Oil/PDMS Phase Separation}

The experimental observation, as shown in Figure 3, verified the validity of the abovementioned thermodynamic analysis. The structure of methyl silicone oil is similar to that of PDMS, but the difference lies in the groups of the end groups. In any proportion of the blend solution, methyl silicone oil and PDMS were miscible, as shown in Figure 3a, and no phase separation was observed; therefore, methyl silicone oil and PDMS are completely compatible. 
(a)

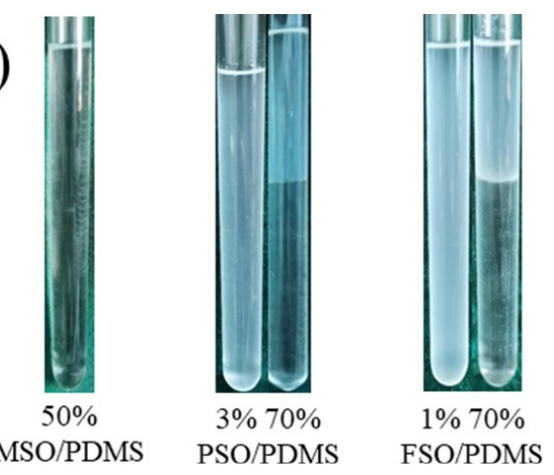

(b)

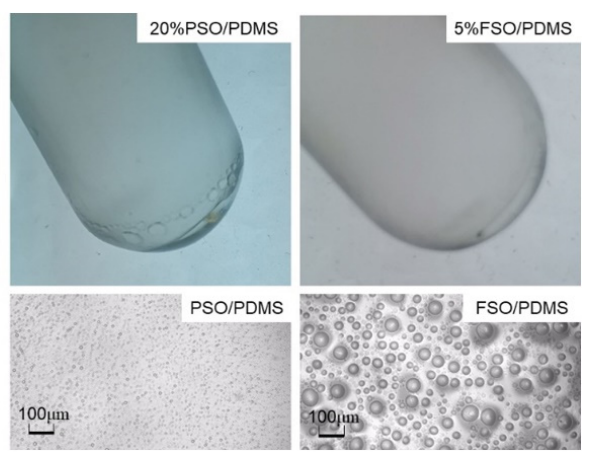

Figure 3. Morphology of different silicone oil/PDMS mixtures: (a) macroscopic images; (b) OM images.

The structural difference between phenyl silicone oil and PDMS mainly comes from the benzene ring on the side chain, which leads to the poor compatibility between phenyl silicone oil and PDMS. When the concentration was $1 \%$, the mixed solution in the test tube was transparent. When the concentration was $2 \%$, it became turbid. When the concentration was 3\%, the mixed solution remained turbid, as shown in Figure 3a. With the increase, precipitated phenyl silicone oil droplets were observed at the bottom at a $20 \%$ concentration, as shown in Figure 3b, and an obvious stratification phenomenon was observed when the PSO concentration was up to and over 30\%. After standing for half an hour, the delamination was completed and could maintain stability, as shown in Figure 3a.

There are perfluorinated groups in the side chain of FSO. Due to the polarity of fluorine atoms, the compatibility between FSO and PDMS is very poor. FSO has the worst compatibility, and phase separation even occurs at very low concentrations. As it is shown in Figure 3a, when the concentration is $1 \%$, a turbid mixture can be observed. When the concentration was $5 \%$, as shown in Figure 3b, FSO droplets precipitated at the bottom of the test tube. At up to $10 \%$, the phenomenon of delamination was obviously observed. However, because of the high viscosity of FSO, the intermolecular movement is slow. Although it can be layered at a low concentration, it often takes several hours to complete the delamination.

The silicone oil droplets changed when increasing silicone oil's concentration in PDMS, which is shown, respectively, for PSO/PDMS and FSO/PDMS mixtures in Figures 4 and 5. The immiscible silicone oil droplets can be seen clearly in the blend solution under the microscope. Furthermore, the size of the droplets became larger after increasing the concentration of silicone oil. The size of FSO droplets was larger than that of PSO droplets, due to the weaker compatibility of FSO with PDMS.

(a)
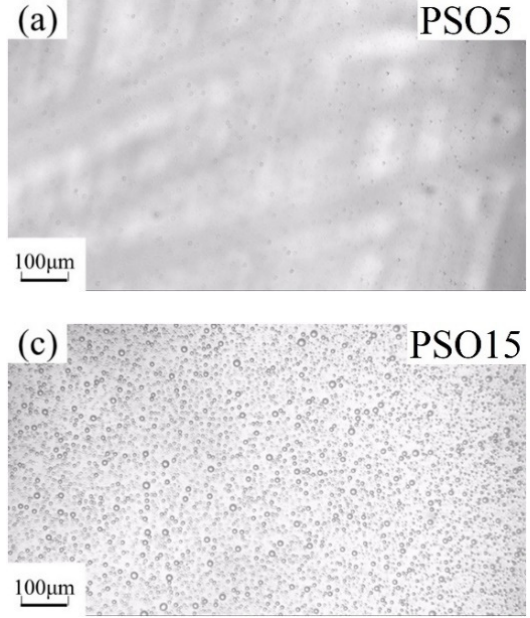
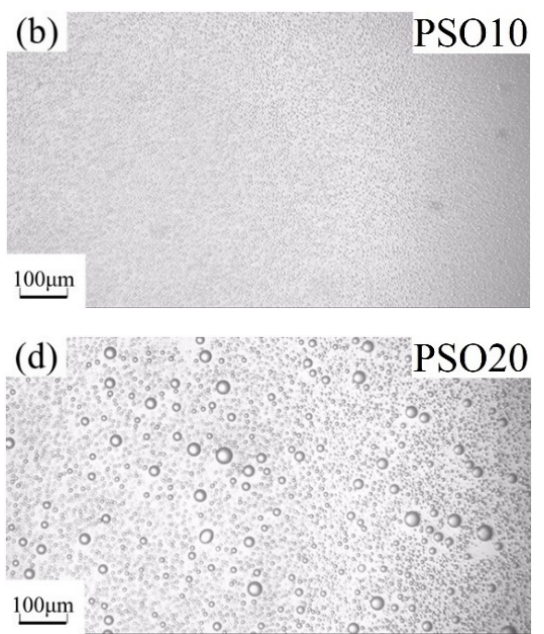

Figure 4. OM images of PSO/PDMS mixtures: (a) PSO5, (b) PSO10, (c) PSO15, (d) PSO20. 
(a)

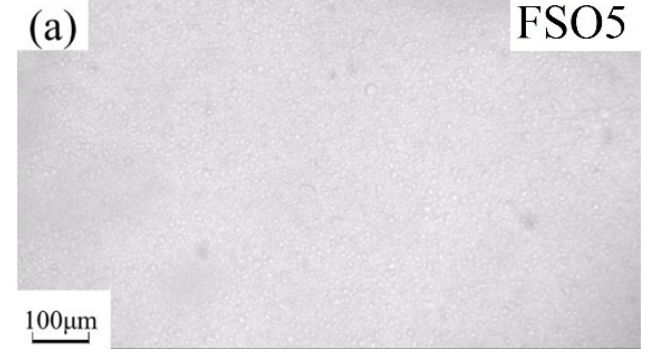

(c)

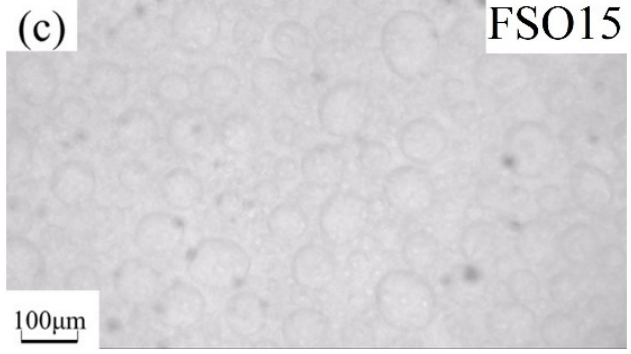

(b)

FSO10

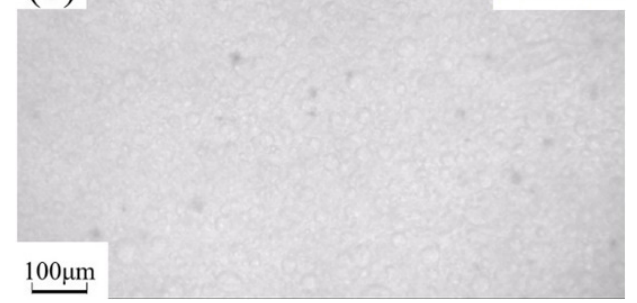

(d)

FSO20

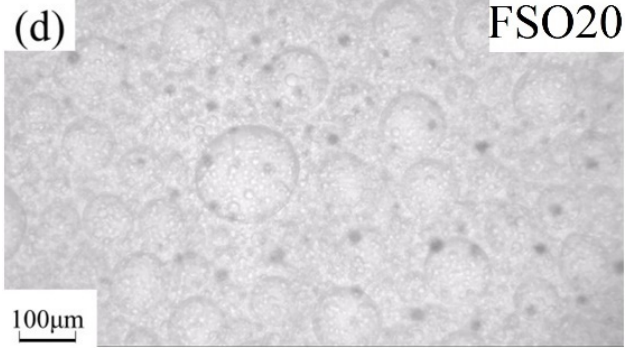

$\underline{100 \mu \mathrm{m}}$

Figure 5. OM images of FSO/PDMS mixtures: (a) FSO5, (b) FSO10, (c) FSO15, (d) FSO20.

Further quantitative analysis shows that the proportion of layered silicone oil in the total volume of the mixture increases linearly with the increase in the silicone oil concentration, as shown in Figure 6, and the limit proportion of the two types of PSO and FSO tends to be the same.

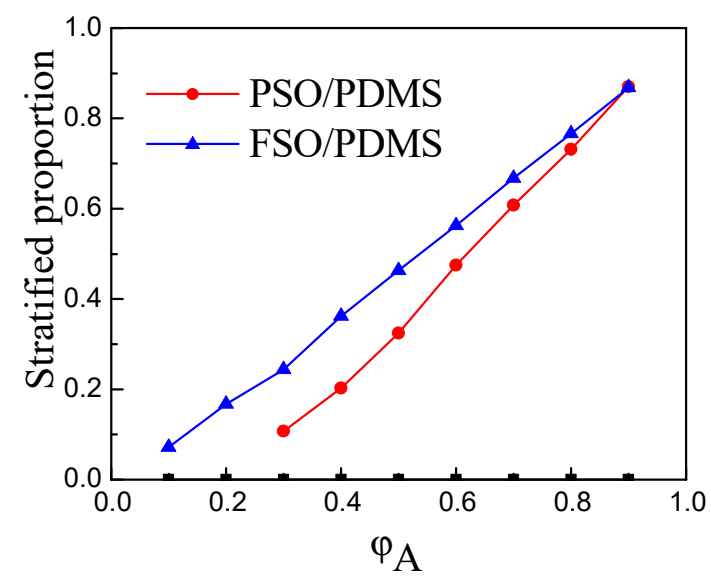

Figure 6. Concentration of silicone oil and stratified proportion.

\subsection{Fracture Microstructure of the Cured Coatings}

The compatibility of silicone oil and polydimethylsiloxane and the degree of phase separation in the mixed solution will inevitably affect the microstructure of the cured coating. As it is shown in Figure $7 \mathrm{a}, \mathrm{d}, \mathrm{g}, \mathrm{j}$, the MSO droplets produced by phase separation cannot be found in the fracture of samples MSO-Z5, MSO-Z10, MSO-Z15 and MSO-Z20. The solubility of MSO and PDMS is excellent, and they can dissolve each other in any proportion. In the liquid state, the phase separation of MSO dissolved in PDMS still did not occur during the film-forming process. 

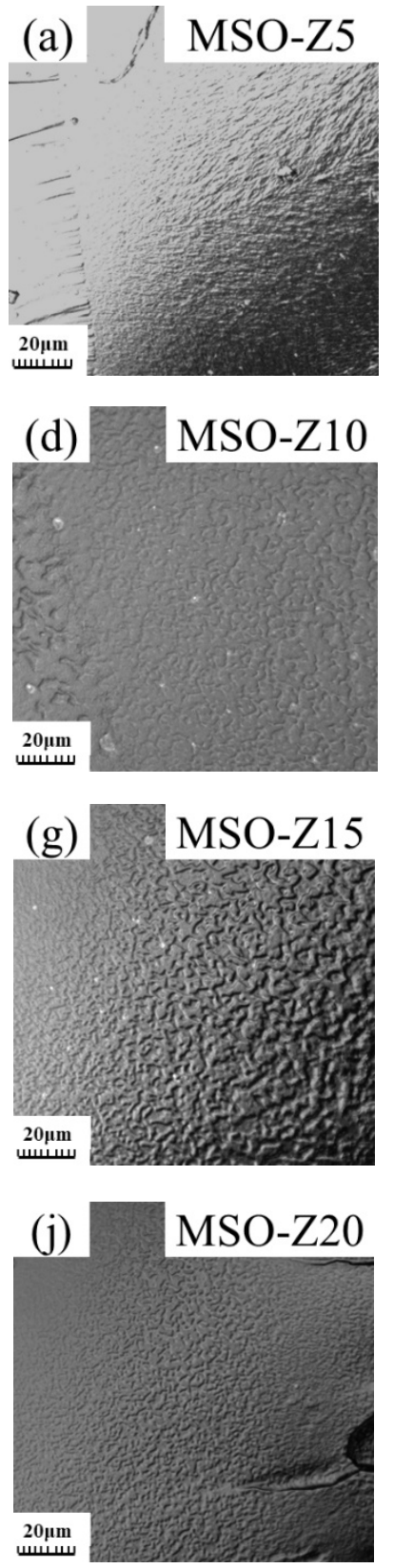
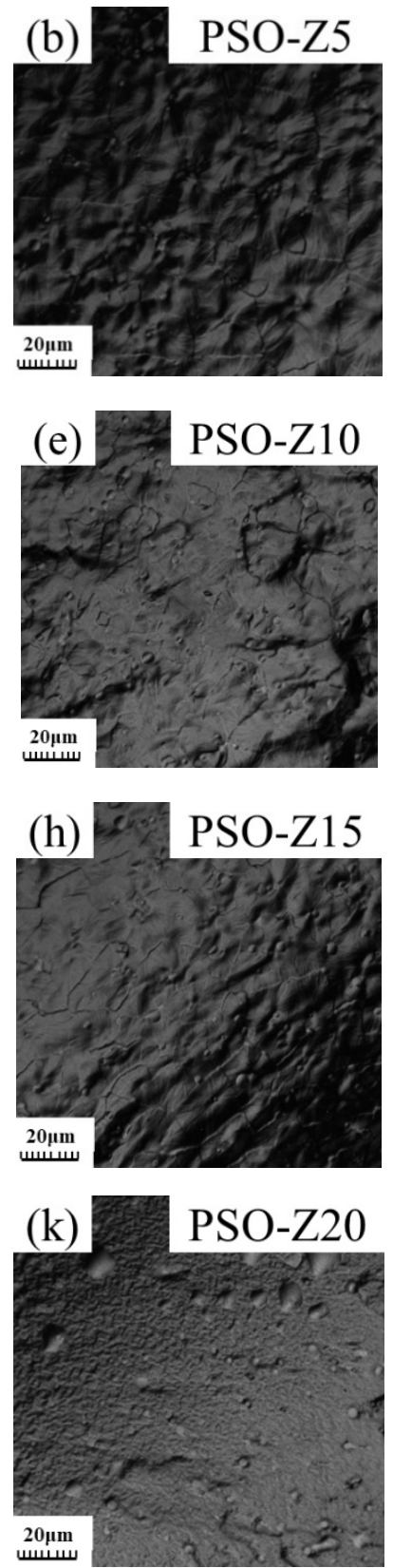
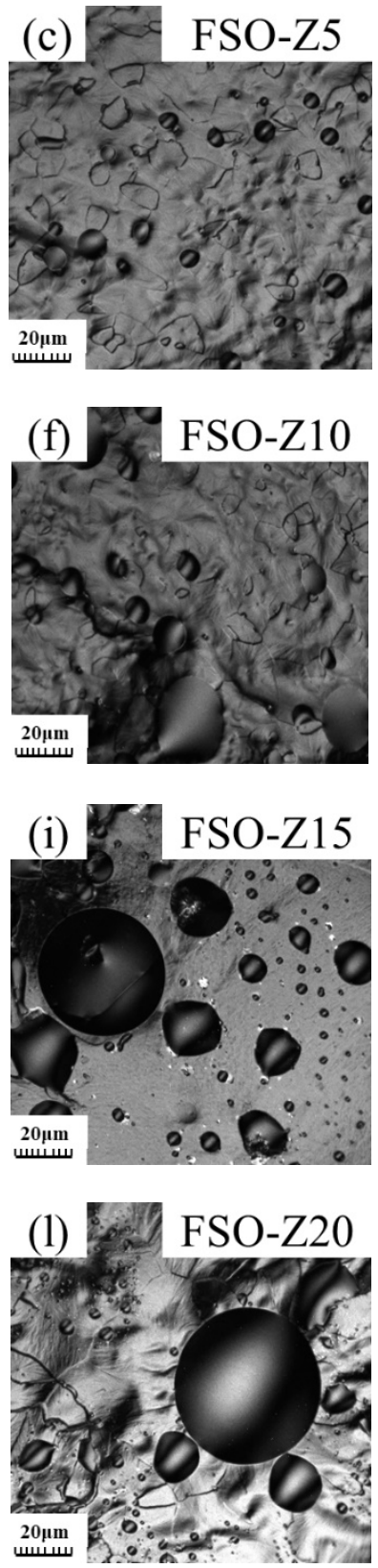

Figure 7. CLSM fracture morphology of different coatings: (a) MSO-Z5, (b)PSO-Z5, (c) FSO-Z5, (d)MSO-Z10, (e) PSO-Z10, (f) FSO-Z10, (g) MSO-Z15, (h) PSO-Z15, (i) FSO-Z15, (j) MSO-Z20, (k) PSO-Z20, (1) FSO-Z20.

The trace of PSO droplets on the fracture surface of the coating can be found in Figure $7 \mathrm{~b}, \mathrm{e}, \mathrm{h}, \mathrm{k}$.

The compatibility between FSO and PDMS is very bad. The phase-separated FSO droplets can be seen clearly in Figure 7c,f,i,l. Compared with PSO, FSO has poor miscibility with PDMS and high viscosity, meaning the volume of FSO droplets formed under the same stirring action was large. Therefore, the size of FSO droplets on the fracture is bigger than that of PSO.

When comparing Figure 4 with Figures 5 and 7 , it can be found that when the phaseseparated silicone oil can be observed in the blending solution, the phase-separated silicone oil can also be observed at the fracture. When the volume of phase-separated silicone oil is large, the large and irregular silicone oil droplets can also be observed at the fracture; this 
shows that the free silicone oil droplets in the coating are related to the silicone oil droplets in the blend solution, and the silicone oil droplets in the blend solution will continue to exist in the silicone coating in a free form during the curing process.

\subsection{Leaching Behavior of Silicone Oil on the Coating}

The morphologies of the three types of coatings exposed for 1 month to ambient conditions were observed by OM and CLSM, shown in Figure 8. As it is shown in Figure 8a,c, both MSO and FSO cannot be observed even by CLSM on the coating surface. There are no free silicone oil droplets in the coating, meaning there is no silicone oil precipitation on the coating surface. As MSO is miscible with PDMS in any proportion, phase separation cannot occur between MSO and PDMS. On the contrary, the surface of the FSO/PDMS coating has no leached trace of silicone oil due to the poor compatibility of FSO with PDMS, and to the higher density of FSO. As it is shown in Figure 8b, phase-separated PSO droplets can be observed. The reason is that PSO is partially compatible with PDMS, which can diffuse through capillary action and move to the coating surface. With the increase in the PSO content in PDMS, as shown in Figure 9, not only the coverage area of leached PSO on the coating surface increased, where it increased from $28.09 \%$ for coating PSO-Z5 to $37.23 \%$ for PSO-Z10, $43.36 \%$ for PSO-Z15 and $46.04 \%$ for PSO-Z20, but also the size of the leached PSO droplets increased.
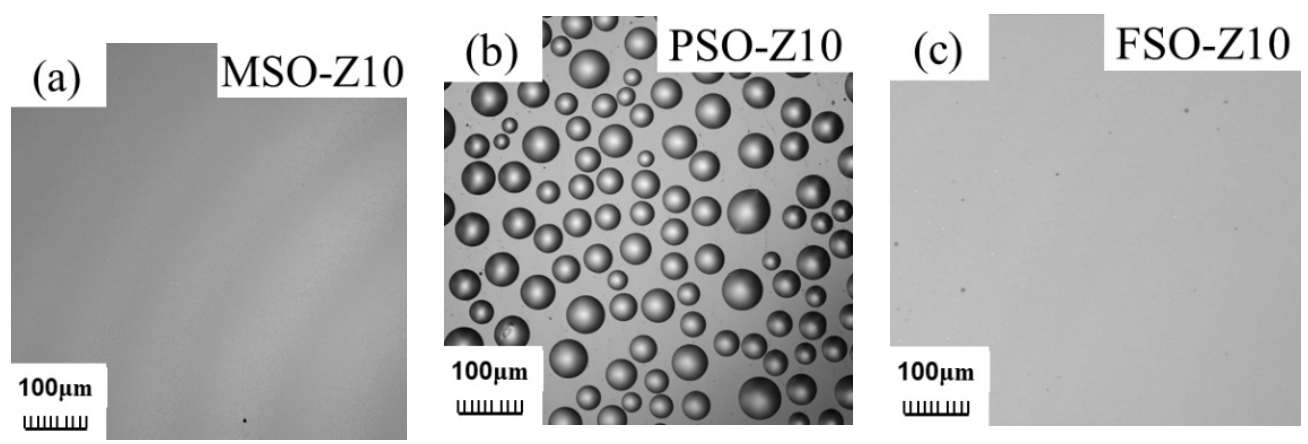

Figure 8. CLSM morphology of silicone oil/PDMS coatings: (a) MSO-Z10, (b) PSO-Z10, (c) FSO-Z10.
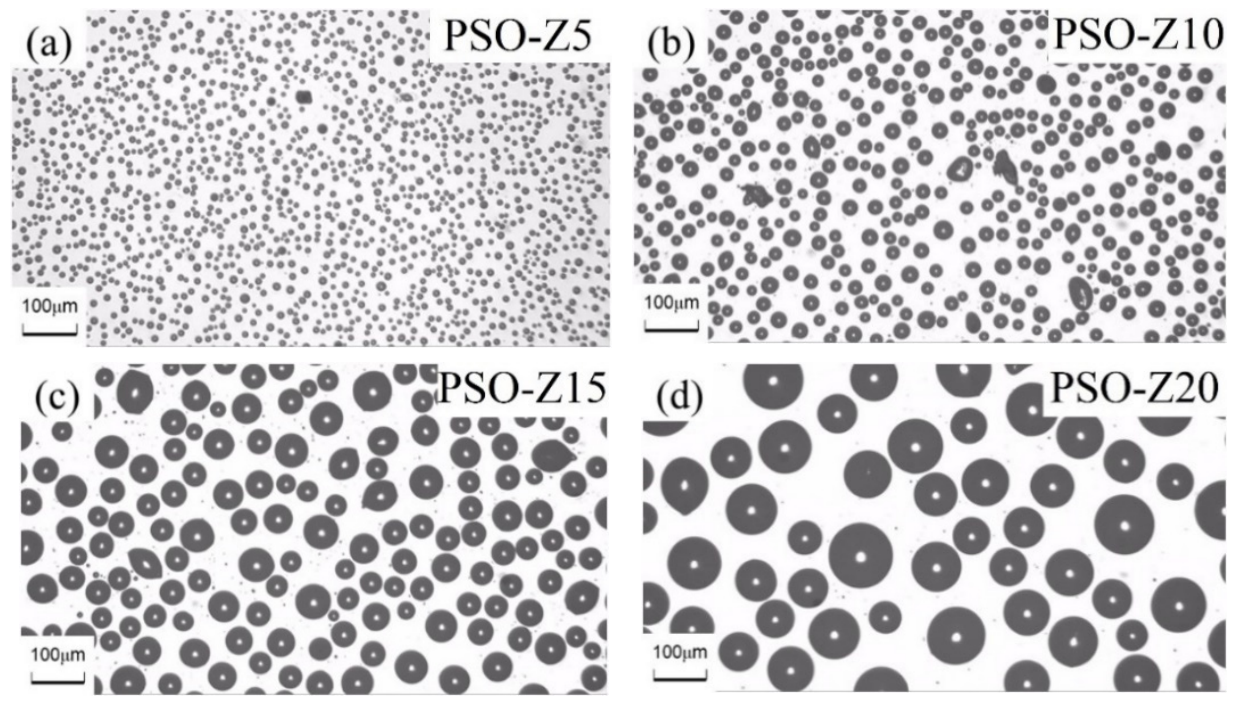

Figure 9. OM morphology of leached PSO droplets on PSO/PDMS coatings: (a) PSO-Z5, (b) PSO-Z10, (c) PSO-Z15, (d) PSO-Z20.

\subsection{Mechanical Properties of the Composite Coatings}

The tensile stress-strain curves of the coatings are shown in Figure 10. For the same silicone oil, the stress-strain curves and tensile behavior of the coatings hardly changed 
when increasing the silicone oil concentration. Compared to the pure PDMS coating, as shown in Figure 10d, the tensile curve of MSO-Z10 is highly coincident with PDMS because methyl silicone oil is dissolved in the silicone coating, and there is no phase separation. However, under the same strain, the tensile stress of PSO-Z10 and FSO-Z10 decreased to some degree due to phase separation, which resulted in silicone oil droplets forming in the coating. The droplets formed in FSO-Z10 are larger and more severe than those in PSO-Z10. Therefore, the decline in the mechanical properties is more serious. In addition, by introducing silicone oil, as shown in Figure 11, the elastic modulus and hardness of the coating decreased to a certain extent respectively with the increase in the silicone oil addition. In conclusion, the lower the compatibility of silicone oil with PDMS, the more silicone oil droplets produced by phase separation during the curing process of the coating, the larger the size of the droplets and the lower the elastic modulus and hardness of the composite coating.
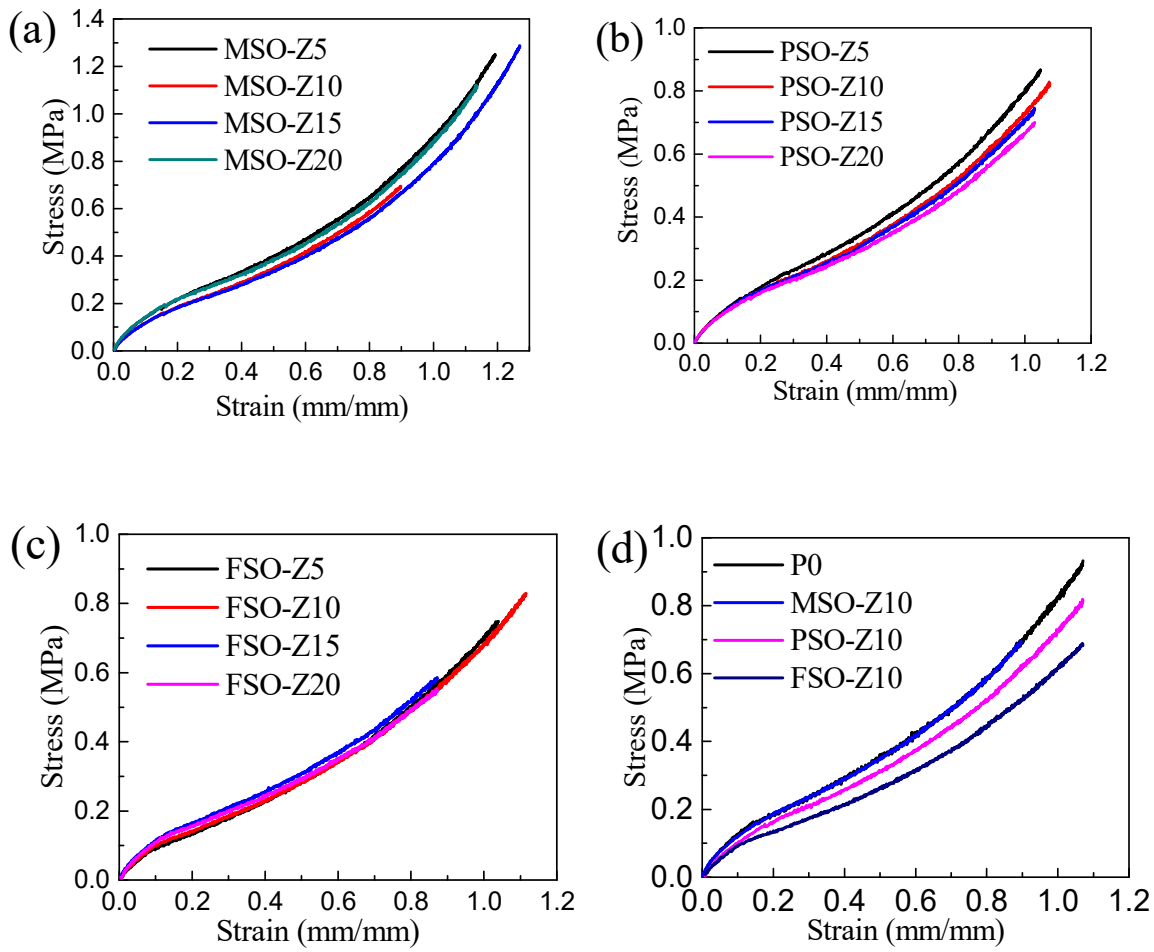

Figure 10. Tensile stress-strain curves of silicone coatings: (a) MSO/PDMS coating, (b) PSO/PDMS coating, (c) FSO/PDMS coating, (d) different silicone oil/PDMS coatings.

(a)

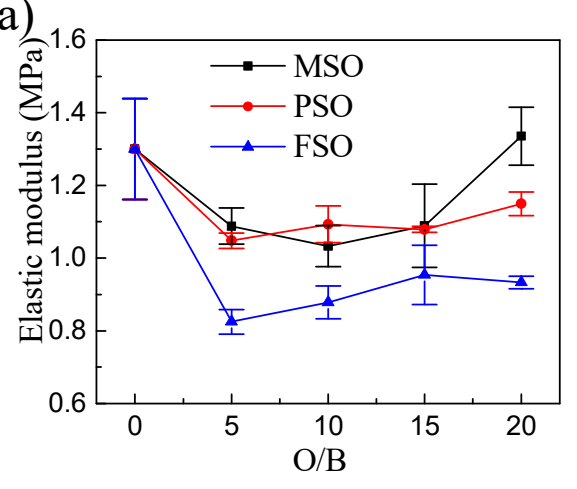

(b)

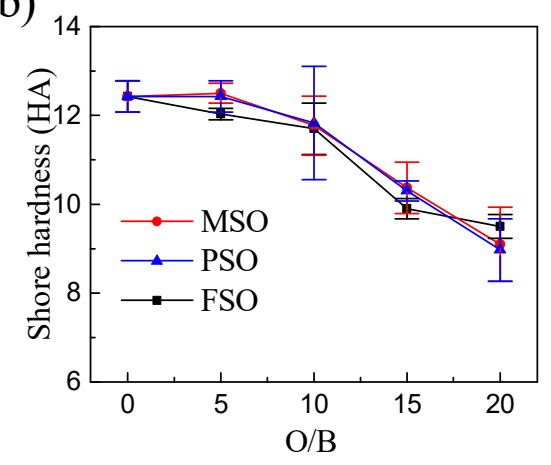

Figure 11. Mechanical properties of coatings change with $\mathrm{O} / \mathrm{B}$ in the coatings of different silicone oils: (a) elastic modulus, (b) hardness. 


\subsection{Surface Energy of the Composite Coatings}

For the coating with MSO, there is no MSO precipitation on the surface. The structure and group of MSO and PDMS are similar. Therefore, it can be seen from Table 2 and Figure 12 that the surface energy increases slightly with the addition of methyl silicone oil. However, the water and diiodomethane contact angle do not change obviously with increasing MSO, which indicates that the addition of MSO has little effect on the surface properties of the coating.

Table 2. The surface properties of different silicone oil/silicone coatings.

\begin{tabular}{cccc}
\hline \multirow{2}{*}{ Sample } & \multicolumn{2}{c}{ Contact Angle $\left(^{\circ}\right)$} & Surface Free Energy $\left(\mathbf{m J} / \mathbf{m}^{\mathbf{2}}\right)$ \\
\cline { 2 - 4 } & Water & Diiodomethane \\
\hline P0 & $108.05 \pm 1.21$ & $72.65 \pm 1.49$ & $21.49 \pm 0.86$ \\
MSO-Z10 & $107.15 \pm 1.09$ & $70.15 \pm 1.52$ & $22.89 \pm 0.86$ \\
PSO-Z10 & $97.10 \pm 0.51$ & $53.85 \pm 1.83$ & $32.39 \pm 1.02$ \\
FSO-Z10 & $108.75 \pm 1.15$ & $68.85 \pm 2.43$ & $23.79 \pm 1.41$ \\
\hline
\end{tabular}
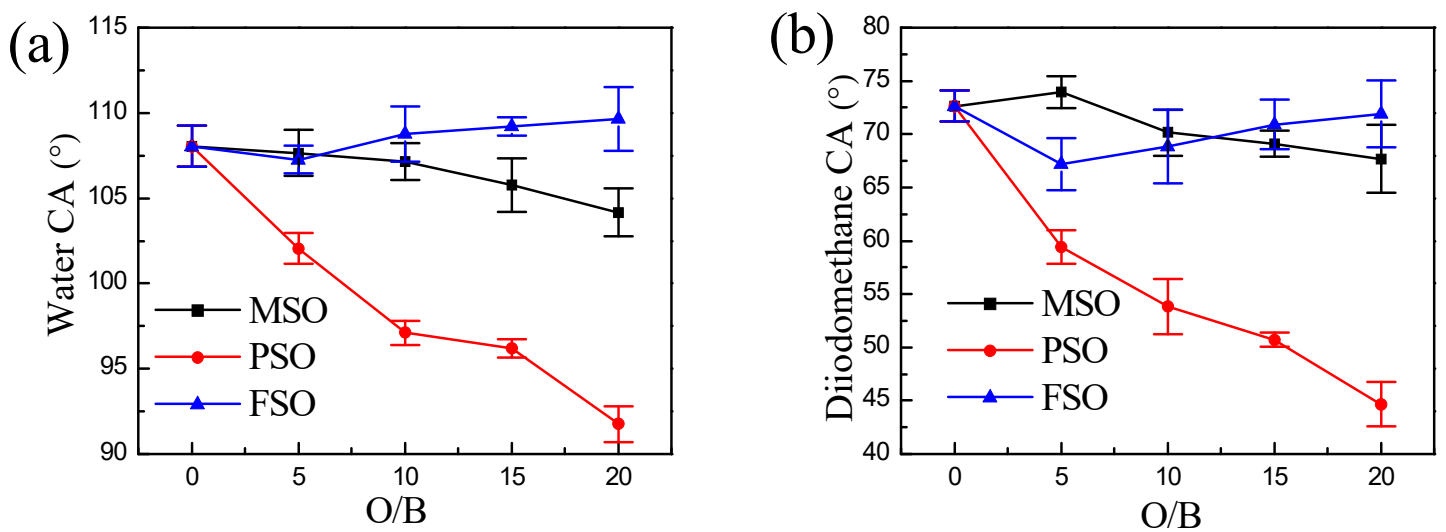

Figure 12. $\mathrm{CA}$ changes with $\mathrm{O} / \mathrm{B}$ in the coatings of different silicone oils, (a) water contact angle and (b) diiodomethane contact angle.

However, a large amount of silicone oil was precipitated on the surface of the PDMS coating with the added PSO. As shown in Figure 12, the higher the amount of PSO, the higher the amount of PSO that is precipitated on the coating. Compared with PDMS, PSO has many phenyls in the side chain, and phenyl has polarity. Therefore, the water contact angle and diiodomethane contact angle of the coating decreased significantly with the increase in PSO, but the surface energy of the coating increased.

The side chain of FSO has a perfluorinated group, which has strong polarity and an amphiphilic property. However, no FSO precipitates on the surface of the coating. Therefore, both the contact angle and surface energy hardly changed with the increase in FSO.

\section{Conclusions}

The phase separation of MSO, PSO and FSO with PDMS blend solutions was consistent with the thermodynamic calculation. When the concentration of PSO and FSO reaches a certain degree, the delamination phenomenon will appear.

The distribution of silicone oil in the blend coating was the same as that in the blend solution. The morphology of silicone oil droplets produced by phase separation in the blend solution was consistent with that observed in the fracture surface of the coating, which indicates that the phase-separated droplets in the blend solution were fixed during the curing process and remained free. 
There was no silicone oil on the surface of the MSO/PDMS coating and FSO/PDMS coating. In contrast, silicone oil could be observed on the surface of the PSO/PDMS coating, and the amount of silicone oil precipitation increased with the increase in the PSO content.

The mechanical properties of the coating decreased with the increase in the silicone oil content. There was no silicone oil on the surface of the MSO/PDMS coating and FSO/PDMS coating after being exposed for 1 month to ambient conditions. The water contact angle, diiodomethane contact angle and surface energy hardly changed. PSO was easy to move onto the surface of the composite coating. The contact angle and surface energy of the coating obviously reduced with increasing PSO content.

Therefore, phenyl silicone oil is the best choice among the three silicone oils to improve the antifouling performance by imitating the behavior of marine organisms secreting mucus. The leaching effect of PSO can be controlled by its content and compatibility with PDMS.

Author Contributions: Conceptualization, Y.J. and Z.Z.; methodology, Y.J., Z.Z. and Y.Q.; software, Y.J. and Z.Z.; validation, Y.J. and Z.Z.; formal analysis, Y.J.; investigation, Z.Z.; resources, Y.Q.; data curation, Y.J.; writing-original draft preparation, Y.J.; writing-review and editing, Z.Z.; visualization, Z.Z. and Y.Q.; supervision, Z.Z.; project administration, Y.Q.; funding acquisition, Z.Z. and Y.Q. All authors have read and agreed to the published version of the manuscript.

Funding: This research is supported by the National Science Foundation of China (51879021).

Data Availability Statement: Data are contained within the article.

Conflicts of Interest: The authors declare no conflict of interest.

\section{References}

1. Callow, J.A.; Callow, M.E. Trends in the development of environmentally friendly fouling-resistant marine coatings. Nat. Commun. 2011, 2, 244. [CrossRef]

2. Dundua, A.; Franzka, S.; Ulbricht, M. Improved Antifouling Properties of Polydimethylsiloxane Films via Formation of Polysiloxane/Polyzwitterion Interpenetrating Networks. Macromol. Rapid Commun. 2016, 37, 2030-2036. [CrossRef]

3. Lejars, M.; Margaillan, A.; Bressy, C. Fouling Release Coatings: A Nontoxic Alternative to Biocidal Antifouling Coatings. Chem. Rev. 2012, 112, 4347-4390. [CrossRef]

4. Yang, W.J.; Neoh, K.G.; Kong, E.T. Polymer brush coatings for combating marine biofouling. Prog. Polym. Sci. 2014, 39, 1017-1042. [CrossRef]

5. Silva, E.R.; Ferreira, O.; Ramalho, P.A. Eco-friendly non-biocide-release coatings for marine biofouling prevention. Sci. Total Environ. 2019, 650, 2499-2511. [CrossRef]

6. Ba, M; Zhang, Z.; Qi, Y. The influence of MWCNTs-OH on the properties of the fouling release coatings based on polydimethylsiloxane with the incorporation of phenylmethylsilicone oil. Prog. Org. Coat. 2019, 130, 132-143. [CrossRef]

7. Nurioglu, A.G.; Esteves, A.C.C.; de With, G. Non-toxic, non-biocide-release antifouling coatings based on molecular structure design for marine applications. J. Mater. Chem. B 2015, 3, 6547-6570. [CrossRef]

8. Stafslien, S.J.; Bahr, J.A.; Feser, J.M. Combinatorial materials research applied to the development of new surface coatings I: A multiwell plate screening method for the high-throughput assessment of bacterial biofilm retention on surfaces. J. Comb. Chem. 2006, 8, 156-162. [CrossRef]

9. Chisholm, B.J.; Webster, D.C.; Bennett, J.C. Combinatorial materials research applied to the development of new surface coatings VII: An automated system for adhesion testing. Rev. Sci. Instrum. 2007, 78, 072213. [CrossRef]

10. Pan, Y.; Zhang, S.P.; Zhou, J.L. Controlling Factors and Progress of Low Surface Energy Silicone Antifouling Coatings. Paint Coat. Ind. 2009, 39, 58-61.

11. Baier, R.E. Surface behaviour of biomaterials: The theta surface for biocompatibility. J. Mater. Sci. Mater. Med. 2006, 17, 1057-1062. [CrossRef]

12. Brady, R.F.; Singer, I.L. Mechanical factors favoring release from fouling release coatings. Biofouling 2000, 15, 73-81. [CrossRef]

13. Brady, R.F. Properties which influence marine fouling resistance in polymers containing silicon and fluorine. Prog. Org. Coat. 1999, 35, 31-35. [CrossRef]

14. Stafslien, S.J.; Christianson, D.; Daniels, J. Combinatorial materials research applied to the development of new surface coatings XVI: Fouling-release properties of amphiphilic polysiloxane coatings. Biofouling 2015, 31, 135-149. [CrossRef]

15. Ware, C.S.; Smith-Palmer, T.; Peppou-Chapman, S. Marine Antifouling Behavior of Lubricant-Infused Nanowrinkled Polymeric Surfaces. ACS Appl. Mater. Interfaces 2018, 10, 4173-4182. [CrossRef]

16. Mueller, W.J.; Nowacki, L.J. Ship's Hull Coated with Antifouling Silicone Rubber. U.S. Patent 3,702,778, 14 November 1972.

17. Yonehara, Y.; Nanishi, K. Siloxane Polymer Antifouling Paint Composition Containing Polysiloxanes. U.S. Patent 4,910,252, 20 March 1990. 
18. Masato, K.; Makoto, H.; Toshimitsu, M. Antifoul- ing Coating Composition. EP Patent 1,275,705, 28 March 2000.

19. Takafumi, S. Antifouling Coating Composition and Un-derwater Structure Using the Same. EP Patent 2,143,766, 13 January 2010.

20. Ba, M.; Zhang, Z.P.; Qi, Y.H. The Dispersion Tolerance of Micro/Nano Particle in Polydimethylsiloxane and Its Influence on the Properties of Fouling Release Coatings Based on Polydimethylsiloxane. Coatings 2017, 7, 7070107.

21. Chang, J.F.; He, X.Y.; Bai, X.Q. The impact of hydrodynamic shear force on adhesion morphology and biofilm conformation of Bacillus sp. Ocean Eng. 2020, 197, 106860. [CrossRef]

22. Stein, J.; Truby, K.; Wood, C.D. Silicone foul release coatings: Effect of the interaction of oil and coating functionalities on the magnitude of macrofouling attachment strengths. Biofouling 2003, 19, 71-82. [CrossRef]

23. Chen, R.R.; Li, Y.K.; Tang, L. Synthesis of zinc-based acrylate copolymers and their marine antifouling application. RSC Adv. 2017, 7, 40020-40027. [CrossRef]

24. Eduok, U.; Faye, O.; Szpunar, J. Recent developments and applications of protective silicone coatings: A review of PDMS functional materials. Prog. Org. Coat. 2017, 111, 124-163. [CrossRef]

25. Milne, A. Anti-Fouling Marine Compositions. U.S. Patent 4,025,693, 24 May 1977.

26. Hoipkemeier-Wilson, L.; Schumacher, J.; Carman, M. Antifouling potential of lubricious, micro-engineered, PDMS elastomers against zoospores of the green fouling alga Ulva (Enteromorpha). Biofouling 2004, 20, 53-63. [CrossRef]

27. Truby, K.; Wood, C.; Stein, J. Evaluation of the performance enhancement of silicone biofouling-release coatings by oil incorporation. Biofouling 2000, 15, 141-150. [CrossRef] [PubMed]

28. Shivapooja, P.; Cao, C.Y.; Orihuela, B. Incorporation of silicone oil into elastomers enhances barnacle detachment by active surface strain. Biofouling 2016, 32, 1017-1028. [CrossRef] [PubMed]

29. Loriot, M.; Linossier, I.; Vallee-Rehel, K. Influence of Biodegradable Polymer Properties on Antifouling Paints Activity. Polymers 2017, 9, 36. [CrossRef] [PubMed]

30. Galhenage, T.P.; Hoffman, D.; Silbert, S.D. Fouling-Release Performance of Silicone Oil-Modified Siloxane-Polyurethane Coatings. ACS Appl. Mater. Interfaces 2016, 8, 29025-29036. [CrossRef]

31. Oliva, M.; Martinelli, E.; Galli, G. PDMS-based films containing surface-active amphiphilic block copolymers to combat fouling from barnacles B. amphitrite and B. improvises. Polymer 2017, 108, 476-482. [CrossRef]

32. Wang, X.T.; Olsen, S.M.; Martinez, E.A. Drag resistance of ship hulls: Effects of surface roughness of newly applied fouling control coatings, coating water absorption, and welding seams. J. Coat. Technol. Res. 2018, 15, 657-669. [CrossRef]

33. Ba, M.; Zhang, Z.P.; Qi, Y.H. Fouling Release Coatings Based on Polydimethylsiloxane with the Incorporation of Phenylmethylsilicone Oil. Coatings 2018, 8, 153. [CrossRef]

34. ISO 37:2005-Rubber, Vulcanized or Thermoplastic-Determination of Tensile Stress-Strain Properties; International Organization for Standardization: Geneva, Switzerland, 2005.

35. Hua, Y.Q.; Jin, R.G. Polymer Solution. Polymer Physics, 4nd ed.; Yang, Q., Li, Y., Eds.; Chemical Industry Publishing Car: Beijing, China, 2013; Volume 3, pp. 101-103.

36. Liu, D.Z.; Wang, Z.Q. Thermodynamics of Polymer Solution. Solubility Parameter and Its Application in Coating Industry, 1st ed.; Yang, C.X., Liu, Z.H., Eds.; Ocean Press: Beijing, China, 2008; Volume 6, pp. 75-76. 